\title{
A INFLUÊNCIA DO NÍVEL SOCIOECONÔMICO NA APRENDIZAGEM DA LEITURA
}

\author{
The influence of the socioeconomic level on learning to read
}

Thalita Rodrigues Silva - FCMMG/Brasil

Júlia Beatriz Lopes Silva - UFMG/Brasil

\begin{abstract}
RESUMO: Os dados do IBGE de 2007 demonstram que 2,4 milhões $(8,4 \%)$ de crianças entre 07 e 14 anos não sabiam ler e escrever, sendo que $87,2 \%$ delas frequentavam a escola. O IBGE também aponta que somente 3,8\% dos estudantes de famílias pobres estudam em escolas particulares. O tipo de escola está bastante associado ao nível socioeconômico familiar, embora não se restrinja a ele, fator que prediz em grande medida o desempenho em leitura. $\mathrm{O}$ objetivo deste estudo foi sistematizar evidências sobre a influência do nível socioeconômico na aprendizagem da leitura. A busca de artigos foi realizada nas bases Scielo e Google acadêmico. As publicações analisadas descrevem sobre as influências na aprendizagem da criança, relacionando-as principalmente com o nível socioeconômico das mesmas, ressaltando também outras influências na aprendizagem da criança. As publicações também destacam a função da família e da escola no processo de aprendizagem. A aprendizagem é um processo complexo e que está relacionado a diversos fatores: ambientais, biológicos, cognitivos e psicológicos. O resultado desse artigo contribuirá para compreensão da dificuldade de aprendizagem da criança, podendo pensar em políticas e programas a serem oferecidos para esta população a fim de minimizar as dificuldades de aprendizagem destas crianças e contribuir no processo de leitura.
\end{abstract}

Palavras-chave: Aprendizagem de leitura. Aprendizado. Fatores socioeconômicos.

ABSTRACT: According to IBGE data from 2007, 2.4 million (8.4\%) of children between the ages of 7 and 14 did not know how to read and write, with $87.2 \%$ of them attending school. IBGE also points out that only $3.8 \%$ of students from poor families study in private schools. The type of school is strongly associated with the family socioeconomic level, although it is not restricted to it, a factor that largely predicts reading performance. The goal of this study was to show evidence about the influence of the socioeconomic level on reading learning. The articles search wascarried out at the Scielo and Google academic bases. The analyzed publications describe the influences on the child's learning, relating them mainly to the socioeconomic level of the children, also highlighting other influences on the child's learning. They also stand out the role of the family and school in the learning process. Learning is a complex system that isrelated to several factors: environmental, biological, cognitive and psychological. The result of this article will contribute to an understanding of the child's learning difficulties, being able to think about policies and programs to be offered to this population in order to minimize the learning difficulties of these children and contribute to the process of reading.

Keywords: Learning of reading. Learning. Socioeconomic factors.

\section{INTRODUÇÃO}

Educação, Psicologia e Interfaces, Volume 2, Número 2, p. 7-28, Maio/Agosto, 2018.

ISSN: 2594-5343. DOI: https://doi.org/10.37444/issn-2594-5343.v2i2.93 
A escola tem a tarefa de ensinar a ler bem, pois bons leitores são capazes de buscar novos conhecimentos sozinhos. A leitura é um fator de grande importância para o ser humano, pois é através dela que se ganha autonomia, independência e liberdade, a prática da leitura melhora o vocabulário, o raciocínio e a interpretação.

Diante da importância da leitura para o ser humano, esta se torna uma grande preocupação para os educadores, pois, em 2007, a pesquisa do IBGE apontou que, entre as 28,3 milhões de crianças de 7 a 14 anos, que, pela idade, já teriam passado pelo processo de alfabetização, 2,4 milhões $(8,4 \%)$ não sabiam ler e escrever, sendo que $87,2 \%$ delas frequentavam estabelecimentos de ensino (IBGE, 2008).

Em 2012, o Programa Internacional de Avaliação (PISA) de alunos da Organização para Cooperação e Desenvolvimento Econômico (OCDE) indicou uma redução de desempenho para leitura em relação à avaliação anterior, passou de 412 para 410 pontos. Atualmente, o Brasil ocupa a $55^{\mathrm{a}}$ posição de um ranking dentre as 65 economias avaliadas, com 86 pontos a menos da média encontrada pela OCDE (INEP, 2015). Em análise qualitativa do desempenho verificou-se que os alunos brasileiros têm dificuldade de compreensão e não conseguem deduzir informações dos textos lidos (LIMA, 2016).

O Indicador de Alfabetismo Funcional (INAF) de 2011/2012 aponta que menos de um terço dos brasileiros são capazes de ler, compreender e interpretar textos longos e de realizar outras tarefas que exigem domínio de leitura e escrita (CORSO et. al., 2013).

Diante destes dados alarmantes, o presente estudo tem o objetivo de sistematizar evidências sobre a influência do nível socioeconômico na aprendizagem da leitura. Assim, investigando os fatores psicossociais, socioeconômicos e familiares que interferem na aprendizagem da leitura, diante do pressuposto de que a leitura é um processo complexo, relacionado com diversos fatores: ambientais, biológicos, cognitivos e psicológicos.

O texto está estruturado em seis seções: na primeira que é a introdução, a segunda apresenta a metodologia, na terceira busca-se referências acerca de algumas definições teóricas a respeito do processo de leitura, na quarta, busca-se definir os fatores que interferem na aprendizagem da leitura, na quinta seção busca-se descrever sobre a influência da família no aprendizado escolar, na seção seguinte busca-se referências acerca dos fatores psicossociais relacionados à aprendizagem, na sétima seção busca-se 
compreender a importância da escola para o aprendizado da criança. E na sequência busca-se uma correlação entre o nível socioeconômico (NSE) e funções executivas (FE) e por último apresentou as considerações finais.

\section{MATERIAL E MÉTODO}

Este estudo teve como objetivo analisar a produção cientifica a cerca da influência do nível socioeconômico na aprendizagem da leitura. Para isso, realizou-se uma revisão sistemática de literatura, com os artigos sendo pesquisados nas bases de dados Scielo e Google acadêmico, a partir dos descritores aprendizagem, leitura e nível socioeconômico.

Foram selecionados artigos publicados em inglês e português, entre 1995 a 2016. A amostra constituiu-se de artigos, livros e dissertações de mestrado. As publicações analisadas descrevem sobre as influências na aprendizagem da criança, relacionando-as principalmente com o nível socioeconômico das mesmas, ressaltando também outras influências na aprendizagem da criança. As publicações também destacam a função da família e da escola no processo de aprendizagem.

\section{O PROCESSO DA LEITURA}

A leitura é uma atividade complexa que envolve a intervenção de vários mecanismos de comportamento: Identificação de letras, acesso ao significado, integração sintática e semântica. Para compreender o que é lido é preciso ter acesso ao léxico automatizado e habilidades cognitivo-linguísticas mais complexas. Como o aprendizado da leitura é um processo continuo, espera-se que a criança, aos poucos, adquira mecanismos que permitam a junção da palavra escrita, com o seu conhecimento prévio em relação a essa palavra. Assim a criança é capaz de compreender e fazer inferência sobre o que é lido (PICCOLO, 2010).

Sternberg e Grigorenko (2000, apud CORSO et al. 2013) identificam cinco etapas no processo de desenvolvimento da leitura:

1. Reconhecimento da palavra por pista visual: Uma fase de pré-leitura (entre os dois e cinco anos de idade);

2. Reconhecimento da palavra por pista fonológica (entre os cinco e seis anos de idade ou no primeiro ano de escolaridade); 
3. Reconhecimento controlado da palavra: Uso pleno de pistas fonológicas e ortográficas (entre os seis e sete anos de idade, ou no segundo ano de escolaridade);

4. Reconhecimento automático da palavra: $\mathrm{O}$ reconhecimento é rápido e preciso, e não envolve esforço consciente (as crianças podem diferir no grau e no tempo em que automatizam o reconhecimento de palavras);

5. Leitura com estratégias: Com foco no texto, a criança faz uso de habilidades metacognitivas específicas (como ajuste da leitura à dificuldade do material, e utilização de conhecimento prévio) que a ajudam a compreender o que foi lido na sentença ou no parágrafo.

Porém muitas crianças não desenvolvem a leitura de forma esperada, apresentando dificuldades para ler. A dificuldade de leitura "caracteriza-se pela dificuldade de compreensão de palavras e frases escritas, apesar das condições sensoriais da criança estarem intactas" (APA, 2012).

O Manual de Diagnóstico e Estatística dos Transtornos Mentais $5^{\text {a }}$ edição (DSM5) apresenta os seguintes critérios diagnósticos para os transtornos de aprendizagem:

- Leitura de palavras de forma imprecisa ou lenta e com esforço (p. ex., lê palavras isoladas em voz alta, de forma incorreta ou lenta e hesitante, frequentemente adivinha palavras, tem dificuldade de soletrá-las).

- Dificuldade para compreender o sentido do que é lido (p. ex., pode ler o texto com precisão, mas não compreende a sequência, as relações, as inferências ou os sentidos mais profundos do que é lido).

- Dificuldades para ortografar (ou escrever ortograficamente) (p. ex., pode adicionar, omitir ou substituir vogais e consoantes).

- Dificuldades com a expressão escrita (p. ex., comete múltiplos erros de gramática ou pontuação nas frases; emprega organização inadequada de parágrafos; expressão escrita das ideias sem clareza).

- Dificuldades para dominar o senso numérico, fatos numéricos ou cálculo (p. ex., entende números, sua magnitude e relações de forma insatisfatória; conta com os dedos para adicionar números de um dígito em vez de lembrar o fato aritmético, como fazem os colegas; perde-se no meio de cálculos aritméticos e pode trocar as operações). 
- Dificuldades no raciocínio (p. ex., tem grave dificuldade em aplicar conceitos, fatos ou operações matemáticas para solucionar problemas quantitativos).

Para diagnosticar transtorno de aprendizagem deve haver pelo menos um dos sintomas listados acima e que tenha persistido por pelo menos 6 meses, apesar das intervenções dirigidas a essas dificuldades. As habilidades acadêmicas afetadas devem estar abaixo do esperado para a idade do indivíduo, causando prejuízo significativo na vida acadêmica, profissional ou nas atividades do cotidiano, que deverão ser confirmadas por meio de medidas de desempenho padronizadas, e por avaliação clínica abrangente.

As dificuldades de aprendizagem iniciam-se durante os anos escolares, mas podem não se manifestar completamente até que as exigências pelas habilidades acadêmicas afetadas excedam as capacidades limitadas do indivíduo (p. ex., em testes cronometrados, em leitura ou escrita de textos complexos longos e com prazo curto, em alta sobrecarga de exigências acadêmicas).

As dificuldades de aprendizagem não podem ser explicadas por deficiências intelectuais, acuidade visual ou auditiva não corrigida, outros transtornos mentais ou neurológicos, adversidade psicossocial, falta de proficiência na língua de instrução acadêmica ou instrução educacional inadequada.

No DSM-5 o transtorno de aprendizagem com prejuízo em leitura é caracterizado pela dificuldade na leitura de palavras, prejuízo na velocidade ou fluência de leitura e prejuízo na compreensão da leitura. A dislexia é um termo alternativo usado para um padrão de dificuldade de aprendizagem caracterizado por problemas no reconhecimento preciso ou fluente de palavras, problemas de decodificação e dificuldades de ortografia.

\section{FATORES QUE INTERFEREM NA APRENDIZAGEM DA LEITURA}

Há várias divergências sobre o fracasso escolar, Angelucci et al. (2004) descrevem como um problema essencialmente psíquico (do aluno), ou seja, que o fracasso escolar se deve a prejuízos da capacidade intelectual do aluno, decorrentes de problemas emocionais, não se trata de crianças com nível intelectual baixo por carência cultural, mas de uma inibição intelectual causada por dificuldades emocionais (ansiedade, dificuldade de atenção, dependência, agressividade, etc.) adquiridas por relações familiares patologizantes. 
Outra perspectiva encontrada descreve o fracasso escolar como um problema técnico do professor, que usa técnicas de ensino inadequadas ou não domina a técnica correta (ANGELUCCI et al., 2004).

Há também, a visão do fracasso escolar como um problema institucional da escola, que reproduz a estrutura social. E por último, o fracasso escolar é visto como uma questão política, da relação de poder estabelecida quando a escola trabalha com a cultura da classe dominante (ANGELUCCI et al., 2004). Outros autores definem o fracasso escolar como um fenômeno multidimensional, com vários fatores que interferem na aprendizagem (social, cultural, familiar, escolar e individual) (MARCHESI; GIL, 2004).

No Brasil, a literatura sobre a caracterização cognitiva das crianças de mais classes baixas, categoria D e E, que explica a dificuldade no aprendizado da leitura e da escrita, confunde-se com a polêmica discussão sobre o fracasso escolar na escola pública. A revisão dessa literatura mostra as conflitantes abordagens da privação cultural (propondo que as crianças de ambientes menos favorecidos têm déficits e por isso fracassam) e da diferença cultural (propondo que não há déficits, e sim diferenças, que a escola é quem produz o fracasso) (CORSO, 1993). Ou seja:

O foco, entretanto, incide nas relações de poder estabelecidas no interior da instituição escolar, mais especificamente na violência praticada pela escola ao estruturar-se com base na cultura dominante e não reconhecer - e, portanto, desvalorizar - a cultura popular (ANGELUCCI et al., 2004, p 13).

Independentemente do foco ou interpretação, podemos observar que no Brasil as escolas públicas recebem mais alunos de classes mais baixas, e as escolas particulares normalmente recebem alunos das classes média e alta (CORSO et al., 2013). O tipo de escola está bastante associado ao NSE familiar, embora não se restrinja a ele, fator que prediz em grande medida o desempenho em leitura (CORSO et al., 2013).

Dados do IBGE apontam que 8,6\% dos estudantes do ensino médio matriculados nas escolas da rede pública pertencem a famílias com renda per capita na faixa dos $20 \%$ mais ricos do país. Somente 3,8\% dos estudantes de famílias pobres estudam em escolas particulares. Na rede privada, 52,3\% dos estudantes pertencem à faixa de renda mais rica (IBGE, 2012).

Podemos constatar também que os resultados das escolas privadas são superiores as públicas. Corso et al. (2013) avaliaram alunos de quarta a sexta série, de escolas 
públicas e privadas, utilizaram os testes Leitura de palavras isoladas e compreensão textual (questionário e reconto). Investigaram também dissociações entre as habilidades de reconhecer palavras e de compreender textos. O tipo de escola apresentou um efeito principal sobre a leitura de palavras e sobre o reconto. $\mathrm{O}$ fator tipo de escola mostrou ter um efeito sobre Leitura de Palavras Isoladas (LPI), sendo o desempenho dos alunos de escolas públicas significativamente inferior. $\mathrm{O}$ mesmo aconteceu em relação à compreensão de texto medida pelo reconto, tanto quando se usou como variável dependente a categoria do reconto, como quando se tomou a porcentagem da cadeia principal da história. Complementando as análises que mostram o impacto do tipo de escola sobre a leitura, a identificação de grupos de leitores conforme as associações e dissociações nas tarefas de Leitura de Palavras Isoladas (LPI) e Compreensão Leitora (CL) ainda permite verificar que dentro do grupo de maus leitores e de maus compreendedores a concentração de crianças nas escolas públicas é maior. Ao mesmo tempo, o número de leitores proficientes nas escolas privadas é quase o dobro do número de bons leitores em escolas públicas.

O NSE é um fator que permite a identificação de outros fatores, que interferem no desenvolvimento da alfabetização (KIRBY; HOGAN, 2008), como o nível de letramento do ambiente familiar, ou seja, a prática da leitura e escrita, são variáveis que vem sendo usada em estudos sobre os fatores socioeconômicos relacionados com o sucesso na alfabetização.

A habilidade de leitura é uma função multifatorial. Aaron (1995) propôs um modelo de leitura do qual teria influência de fatores ambientais (ecológicos), psicológicos e cognitivos, o modelo propõe que uma criança pode não atingir níveis satisfatórios de leitura por interferências de qualquer componente destes três domínios (LIMA, 2016).

Lima (2016), cita que o desenvolvimento da linguagem escrita das crianças depende da interação de uma série de fatores nos seguintes domínios:

- Domínio cognitivo-linguístico: Reconhecimento de palavras e compreensão de leitura textual e outras habilidades relacionadas à leitura, por exemplo, a memória, atenção, funções executivas e o processamento.

- Domínio psicológico e comportamental: Aspectos como motivação e interesse, lócus de controle, estilos de aprendizagem, expectativa dos professores e diferenças de gênero. 
- Domínio ambiental: Aspectos do ambiente doméstico e da cultura, o envolvimento dos pais, o ambiente de sala de aula e o uso de dialetos.

Segundo Lima (2016), dentre os preditores ambientais do desempenho de leitura das crianças, destaca-se o NSE - índice geralmente composto por renda familiar, escolaridade e ocupação dos pais (MCLOYD, 1998) - e exposição à leitura no ambiente doméstico. Número de livros em casa e estresse parental foram significativamente relacionadas ao desempenho em leitura da criança (AIKENS; BARBARIN, 2008).

Piccolo et al. (2012) realizaram um estudo longitudinal e pesquisaram a contribuição de fatores psicossociais para o desempenho em leitura de crianças. Na qual participaram 59 mães e seus filhos aos 4 meses, 2, 5-6 e 9-11 anos. Correlacionou-se o desempenho em leitura das crianças a variáveis psicossociais e encontraram relação entre sintomas psiquiátricos maternos e a leitura, relação entre o número de familiares que moram com a criança (menor desempenho da criança em leitura associado a famílias mais numerosas) e renda familiar. Concluindo assim, que o desempenho em leitura correlaciona-se a fatores psicossociais.

Enricone e Salles (2011) através de entrevista semiestruturada com 29 familiares de dois grupos de alunos de $2^{a}$ série do ensino fundamental. Por meio de avaliação neuropsicológica, os estudantes foram caracterizados em: com dificuldade de leitura e escrita e leitores e escritores competentes. Neste estudo, as autoras observaram que as crianças com dificuldades de leitura e escrita eram de famílias com maior número de filhos, menor renda, sem residência própria e sem transporte próprio na família; apresentavam índice de repetência escolar e história familiar de dificuldade na leitura.

Fletcher et al. (2007) propõe um modelo para transtornos de aprendizagem, incluindo leitura. Este se baseia nas potencialidades e fraquezas em várias habilidades acadêmicas. Propõe três níveis de análise:

- Déficits em habilidades acadêmicas: Reconhecimento de palavras, compreensão de leitura, fluência na leitura, etc.

- Características da criança, incluindo processos cognitivos como consciência fonológica e nomeação seriada rápida e características pessoais como motivação, habilidades sociais ou problemas comportamentais. 
- Influência dos fatores ambientais e neurobiológicos: Fatores ambientais (ecológicos) representam situações sociais e econômicas que envolvem a criança e fatores neurobiológicos influenciam nas habilidades acadêmicas.

Para Sternberg e Grigorenko (2003, apud Piccolo et al., 2012) O desenvolvimento, assim como as alterações, das habilidades de leitura e escrita em crianças estão relacionados a vários fatores (criança, família, escola, sistema educacional, entre outros).

Segundo Piccolo (2012) a leitura é um processo complexo, correlacionados com fatores ambientais, cognitivos e biológicos. Portanto, o sucesso ou fracasso no processo de ensino-aprendizagem da leitura/escrita sofre a influência de fatores biológicos, neuropsicológicos, psicossociais dos contextos familiar e escolar (SALLES et al., 2010).

Mussen (1970) afirma que para a aprendizagem ocorrer de forma satisfatória é preciso que haja uma motivação, uma necessidade ou desejo de aprender e um reforço, uma recompensa. Porém o autor considera que em alguns processos de aprendizagem podem ocorrer através da identificação com o outro, ou seja, uma criança adquire o padrão de comportamento da família se identificando com seus pais, adquire muitas características, pensamentos e sentimentos deles.

Quando se refere a dificuldades de aprendizagem é necessário levar em consideração o contexto em que a criança se encontra. Assim a família e a escola são grandes responsáveis pela determinação dos distúrbios de aprendizagem (FONSECA, 1999).

\section{A INFLUÊNCIA DA FAMÍLIA NO APRENDIZADO ESCOLAR}

Os pais podem de várias maneiras, favorecer ou prejudicar o processo de aprendizagem de seus filhos. Piccolo et al. (2012) aponta que a família pode oferecer apoio para o desenvolvimento escolar das crianças através da participação dos pais na vida escolar do filho, da organização da rotina da casa e interação entre pais e filhos. A família pode ser um suporte para o desenvolvimento cognitivo da criança ao ofertar brinquedos, livros e oferecer oportunidades para a realização de atividades lúdicas e educativas. A família também pode oferecer suporte emocional através de um ambiente acolhedor, um bom relacionamento entre os pais e entre pais e filhos gera motivação para aprendizagem.

A exposição da leitura no ambiente doméstico é importante para o desempenho escolar da criança. As crianças apresentam um melhor desempenho na escola quando suas 
mães leem mais para elas, diante disto podemos notar como o nível de escolaridade da mãe interfere no desempenho acadêmico da criança. Pesquisas afirmam que mães com maior nível de escolaridade são mais propensas a ler para seus filhos do que mães com níveis mais baixos de escolaridade (KUO et al., 2004; SKIBBE et al., 2008; PICCOLO et al., 2012).

Em sua pesquisa, Piccolo et al. (2012) mostraram que quanto maior o número de pessoas na mesma casa, mais as mães apresentavam sintomas depressivos ou outros problemas emocionais (observados a partir do escore do Inventário de Depressão de Beck - BDI na primeira e segunda etapas e no escore do Questionário de Auto-relato - SRQ-20 na segunda etapa). E que mães com problemas emocionais (conforme o escore no SRQ20) passavam menos tempo com o seu filho (terceira etapa do estudo). Assim, as duas condições (saúde mental da mãe e maior número de pessoas que residem com a criança) influenciam no desempenho em leitura de palavras. Os resultados deste estudo apontaram que a renda correlacionou-se com o escore do BDI, com o escore do SRQ-20 e com o número de pessoas que habitavam a mesma casa que a criança. Esses resultados sugerem que mães com melhor NSE tendem a ter menos problemas emocionais e assim são capazes de se envolver em atividades com seus filhos, principalmente quando as famílias são menores. Dos fatores familiares considerados, foi observada correlação negativa entre o escore da mãe no SRQ-20 (primeira etapa) e o total de acertos na leitura de palavras reais irregulares $(\mathrm{r}=-0,278, \mathrm{p}<0,05)$. Tal resultado aponta que, quanto mais problemas emocionais a mãe apresentou, menor foi o desempenho da criança nessa tarefa (PICCOLO et al. 2012).

A saúde emocional da mãe dificulta a sua relação com a criança, pois mães deprimidas tendem a ser mais irritadas, ansiosas, tristes, menos comunicativas, oferecem menos afeto, e punem mais (RADKE-YARROW; ZAHN-WAXLER, 1990; MENDES et al. 2008; PINTO et al. 2009; PICCOLO et al. 2012).

Comportamentos de apoio como elogios, cuidados e carinhos, essenciais para motivar a criança em relação à aprendizagem são menos frequentes. Leitura de histórias para as crianças, outro fator importante para o desenvolvimento da leitura, demanda disponibilidade emocional e de tempo da mãe, por isso são menos frequentes quando as mães estão deprimidas. Quanto mais pessoas moram na mesma casa, menos tempo a mãe tem disponível para se dedicar a criança (DUURSMA et al. 2008; EVANS et al. 2000; 
FOY; MANN, 2003; HANEY; HILL, 2004; KUO et al. 2004; SÉNÉCHAL; LEFREVE, 2002; PICCOLO et al. 2012). Haney e Hill (2004) apontam que pais com baixa escolaridade encontram mais dificuldade para ajudar as crianças nas tarefas escolares, influenciando no desempenho da criança, já que esta precisa da ajuda de outra pessoa mais preparada.

Andrada et al. (2008) realizaram um estudo com crianças catarinenses de cinco e seis anos de idade, de centros públicos de educação infantil, e concluíram que crianças com melhor desempenho escolar eram aquelas que suas mães tinham maior nível de escolaridade e suas famílias recebiam melhores salários.

Assim, entre os fatores ambientais que interferem no aprendizado da leitura, as características do ambiente familiar ligadas ao NSE, tais como renda e nível de escolaridade dos pais, número de moradores na casa, tempo disponível dos pais com a criança, relacionam-se com as características do ambiente escolar ligadas aos aspectos pedagógicos e ao preparo dos professores (CORSO et al., 2013).

Em resumo, podemos dizer que o desempenho da leitura está relacionado à renda familiar, à saúde emocional da mãe e à quantidade de pessoas que moram com a criança.

Existem muitas pesquisas sobre a influência da família no aprendizado escolar, porém há pouca produção científica no Brasil acerca dos fatores psicossociais que influenciam no desenvolvimento da aprendizagem incluindo a dificuldade de leitura, estes se encontram menos definidos e explorados na literatura brasileira.

\section{FATORES PSICOSSOCIAIS RELACIONADOS À APRENDIZAGEM}

O NSE está relacionado a diversos fatores, como escolaridade, condições nutricionais, quantidade e qualidade de estimulação, cuidados médicos, riscos perinatais, ocupação, estilos de interação familiar e social e condições de habitação. (MIRANDA; MUSZKAT, 2004, ENRICONE; SALLES, 2011).

Dentre os fatores psicossociais relacionados à aprendizagem escolar estão os conceitos de autoeficácia, motivação para aprender, crenças, aspectos afetivos, fatores da escola, ambiente de aprendizagem, tipo e intensidade da instrução, características do professor e recursos no ambiente familiar, como capacidade de lidar com os desafios da integração à escola, envolvimento parental com a escolarização do filho, presença de recursos no ambiente físico, práticas educativas e disciplina. Ainda há variáveis socioeconômicas e variáveis sociolinguísticas, como nível de escolaridade, classe 
socioeconômica e instrução específica sobre regras de escrita, estão relacionadas ao desempenho das crianças em leitura e escrita (SALLES et al. 2010).

Segundo Mussen (1970, apud FONSECA, 1999), valores associados às classes e grupos étnicos diferenciam-se na motivação, na personalidade e nas atitudes das crianças, para o autor crianças de classe média tendem a inibir comportamentos agressivos, já as de classe baixa são encorajadas a expressar agressividade sendo mais propensas a brigas e discussões.

Mussen (1970, apud FONSECA, 1999) relata que as classes sociais se distinguem em relação à motivação de realização. Os pais de classe média em geral valorizam a capacidade de realização de seus filhos, recompensando-os com frequência, os pais de classes mais baixas, categorias D e E, não o fazem. Assim, crianças de classe média mostram mais interesses pelos estudos. Segundo o mesmo autor, crianças de classes mais baixas, categorias D e E tem mais predisposição para dificuldades de aprendizagem, consideram a escola tediosa e há mais probabilidade de ocorrer "problemas escolares".

Porém, não podemos afirmar que somente crianças de classe alta tem desempenho melhor na aprendizagem, crianças de classes inferiores podem alcançar as de classe alta, porém requer mais esforço por parte da criança (FONSECA, 1999).

Conforme o mesmo autor, crianças de classe média possuem melhor vocabulário com uma melhor articulação, falam mais corretamente e gramaticalmente e constroem frases mais elaboradas, estas falam mais com os pais, são mais interessadas na linguagem e recebem mais reforço por realizações verbais do que as crianças de classes mais baixas, categorias D e E (FONSECA, 1999).

Outro fator que exerce grande influência na aprendizagem da criança é a escola, a seguir iremos detalhar sobre a importância da escola.

\section{A IMPORTÂNCIA DA ESCOLA}

A escola é importante para promover o equilíbrio na vida da criança, podendo compensar dificuldades que vieram de uma educação familiar deficitária (FONSECA, 1999).

Para Mussen (1970, apud FONSECA, 1999) as experiências na escola podem promover a confiança em si própria, segundo o autor uma criança triste e retraída cujo ambiente familiar é insatisfatório, áspero e autoritário, ao frequentar uma escola com 
professores afetuosos, tolerantes e compreensivos, pode se tornar uma criança feliz. Por outro lado, experiências infelizes na escola podem cessar os efeitos benéficos de uma boa relação familiar, tornando-se uma criança frustrada, agressiva, retraída e desajustada.

A qualidade das relações estabelecidas na escola de educação infantil pode afetar o aprendizado. Crianças menos envolvidas nas atividades de sala de aula, mais dependentes e mais agressivas nos relacionamentos, mostram menor progresso acadêmico.

A escola (ou seja, a qualidade dos professores e os métodos de ensino) tem impacto nas habilidades de leitura e escrita de crianças (LEYBAERT et al., 1997, ENRICONE; SALLES, 2011). Sobretudo nas escolas públicas, o professor precisa estar preparado para lidar com a diversidade de ambientes socioculturais das crianças (CARVALHO, 2000, ENRICONE; SALLES, 2011).

Diante do exposto podemos destacar a importância do fator emocional sobre a aprendizagem da criança. Sendo essencial para a aprendizagem a formação do autoconceito. "Se julgo que sou capaz de aprender, aprenderei bem mais e melhor do que se me julgar incapaz para tal: dependendo do conceito que faço de mim mesmo, será o conceito que terei do mundo ao meu redor" (FONSECA, 1999, p. 31).

Além do fator emocional, outro aspecto extremamente importante para a aprendizagem da criança são as funções cognitivas. A seguir iremos relatar quais os efeitos que o NSE traz para as funções cognitivas, especificamente as funções executivas.

\section{NÍVEL SOCIOECONÔMICO E FUNÇÕES EXECUTIVAS}

Funções executivas (FE) são um conjunto de habilidades cognitivas que estão relacionados com a organização e regulação do comportamento sendo essenciais para realizar tarefas novas e complexas que precisam de adaptação rápida e flexibilidade às mudanças no ambiente. Essas habilidades ajudam o individuo a se comportar adequadamente, a planejar e na resolução de problemas. Nas crianças, as FE são importantes para regular o comportamento e para controlar comportamentos impulsivos (CARDOSO; FONSECA, 2016).

Conforme Dias e Seabra (2013) existem algumas divergências na literatura sobre o tema, porém os principais componentes das FE são (GAZZANIGA et al., 2006; LEZAK et al., 2004; MALOY-DINIZ et al., 2008): 
- Controle inibitório: a capacidade de controlar o comportamento quando ele é inadequado, assim como inibir a atenção a estímulos que não são relevantes no momento.

- Atenção seletiva: a habilidade de selecionar apenas o que será importante para determinada tarefa em dado momento, de focar a atenção e não distrair-se como os diversos estímulos do ambiente.

- Memória de trabalho: a capacidade de manter a informação em mente e também de transformá-la ou integrá-la com outras informações.

- Flexibilidade cognitiva: a capacidade de mudar o foco e de considerar diferentes alternativas; permite que possamos nos adaptar a diferentes contextos e demandas;

- Monitoramento: Uma habilidade metacognitiva que se refere á capacidade de monitorar os próprios processos mentais ou a própria realização para ver se tudo saiu ou esta saindo conforme o previsto.

- Planejamento: a habilidade de elaborar e executar um plano de ação, de "pensar antes" e de estipular os passos necessários para se atingir um objetivo.

Outras habilidades relacionadas ás funções executivas são (DAWWSON; GUARE, 2010; MELTZER, 2010):

- Organização: a competência de sistematizar informações ou materiais necessários á execução da tarefa.

- Priorização: a capacidade de separar ideias principais de detalhes ou de ordenar a informação com base em sua importância relativa para desempenhar determinada tarefa ou para resolver um problema.

- Manejo do tempo: refere-se á capacidade de estimar o tempo disponível para realizar uma tarefa e de alocá-lo adequadamente. Juntamente com a organização e a priorização, esta habilidade é fundamental ao planejamento da ação, integrando essa última competência.

- Atenção sustentada: capacidade do individuo em manter ou sustentar por um período prolongado de tempo a atenção ao estimulo, mantendo assim, uma resposta consistente ao longo de uma atividade continua.

- Iniciação e persistência em direção ao objetivo: a habilidade de iniciar uma tarefa e de seguir em direção a um objetivo, apesar de outras demandas ou de competidores. 
- Regulação emocional: um processo complexo caracterizado pela expressão de emoções de forma controlada. A autorregulação emocional inclui a capacidade de reconhecer e nomear as próprias emoções, mas, sobretudo, de manejar sua intensidade e modular sua expressão de forma adaptativa em consonância com os vários contextos sociais. Para os autores mencionados, manejar as próprias emoções é um ponto relevante para se alcançar um objetivo, bem como controlar e direcionar o próprio comportamento.

Todas essas habilidades ajudam o indivíduo a organizar seu comportamento de acordo com as exigências do ambiente, monitorar e regular sua conduta (DIAS; SEABRA, 2013).

As funções executivas se desenvolvem durante a infância até a adolescência, o final de seu desenvolvimento ocorre entre 16 e 19 anos (ANDERSON, 2002). As funções executivas são consideradas sujeitas à influência de fatores ambientes já que atingem a maturação neurobiológica mais tarde (HACKMAN; FARAH, 2009). O NSE é um fator contextual que influencia o funcionamento executivo (HACKMAN et al., 2010).

No caso de crianças e adolescentes o NSE é mensurado através da renda e escolaridade dos pais e podem interferir no desenvolvimento neuropsicológico, independente do NSE alcançado a posteriori (GOODMAN et al. 2001). Os mecanismos subjacentes aos efeitos do NSE sobre o funcionamento executivo ainda não são claros; porém, evidências indicam envolvimento de fatores pré-natais, ex.: uso de drogas durante a gestação, nutrição, cuidado parental, estresse, tipo e qualidade da estimulação cognitiva no ambiente familiar, entre outros (HACKMAN et al., 2010).

De acordo com Hackman e Farah (2009) e Hackman et al. (2010), o NSE interfere no desenvolvimento das habilidades executivas de controle inibitório e memória de trabalho (executivo central), porém a conclusão é baseada em um número pequeno de estudos.

Sbicigo et al. (2013) realizaram um estudo cujo objetivo foi sistematizar evidências sobre a influência do NSE no desempenho em tarefas de funções executivas. As autoras encontraram em alguns estudos, que a escolaridade dos pais foi o melhor preditor de FE, especialmente de fluência verbal (ARÁN-FILIPPETTI, 2011; ARDILA et al., 2005; NOBLE et al., 2005). O índice de riqueza familiar foi melhor preditor de memória de trabalho e memória para frases que a escolaridade materna (FERNALD et 
al., 2011). Neste mesmo estudo, a escolaridade materna foi melhor preditora de atenção sustentada.

Foi observado que crianças de famílias com maior NSE apresentavam maior desempenho em tarefas de planejamento, flexibilidade cognitiva/flexibilidade da atenção (ARDILA et al., 2005; NOBLE et al., 2005; RHOADES et al., 2011). Crianças brancas e afro-americanas com NSE mais elevado obtiveram melhor resultado em tarefas de flexibilidade cognitiva aos três anos (RHOADES et al. 2011).

A escolaridade dos pais influencia o desempenho em fluência verbal em crianças e adolescentes (ARÁN-FILIPPETTI, 2011; ARDILA et al., 2005; HURKS et al., 2010). Crianças de pais com maior nível de escolaridade apresentaram desempenho superior em fluência verbal semântica - FVS e fluência gráfica não semântica, mas não em fluência verbal fonêmica (ARDILA et al., 2005).

Diante destes dados, é importante que as escolas, principalmente as públicas, estejam atentas sobre a importância das funções executivas para a aprendizagem da criança, visto que há um déficit maior em crianças de NSE mais baixo é imprescindível que as escolas invistam em programas destinados às FE. Atualmente com o crescente desenvolvimento da Neuropsicologia surgiram programas de estimulação das funções executivas especialmente para escolares, tais como: Pence - Programa de estimulação Neuropsicológica da cognição em escolares: ênfase nas funções executivas (CARDOSO; FONSECA, 2016); Cena - Programa de capacitação de educadores sobre neuropsicologia da aprendizagem com ênfase em funções executivas e atenção (PUREZA; FONSECA, 2016); e o Piafex - programa de intervenção em autorregulação e funções executivas (DIAS; SEABRA, 2013).

\section{CONSIDERAÇÕES FINAIS}

O trabalho de revisão de literatura nos proporcionou refletir a respeito da escola como um fator importante para a aprendizagem da criança, a qualidade dos professores e os métodos de ensino tem impacto nas habilidades de leitura e escrita de crianças. A 
escola é importante para promover o equilíbrio na vida da criança, podendo compensar dificuldades que vieram de uma educação familiar deficitária (FONSECA, 1999).

A qualidade das relações estabelecidas na escola de educação infantil pode afetar o aprendizado. Conforme Mussen (1970), citado no tópico “a importância da escola”, é possível compensar déficits oriundos do contexto familiar ao disponibilizar influências positivas ao aluno. Portanto é recomendável que os professores sejam atenciosos, afetuosos e disponíveis para seus alunos, para que estes tenham motivação para a aprendizagem. Que os professores, sobretudo os de escola pública, estejam preparados para lidar com a diversidade de ambientes socioculturais das crianças (CARVALHO, 2000, ENRICONE; SALES, 2011). Além de destacar o quão é importante as escolas públicas serem bem equipadas e organizadas para acolher as crianças de família de baixa renda.

Outro ponto que podemos destacar foi a influência da família na aprendizagem da criança. Piccollo et al. (2012) abordou detalhadamente este assunto no tópico "a influência da família no aprendizado escolar", que através de uma relação familiar saudável é possível minimizar dificuldades de aprendizagem. A família pode oferecer apoio para o desenvolvimento escolar das crianças através da participação dos pais na vida escolar do filho, da organização da rotina da casa e interação entre pais e filhos.

A família pode ser um suporte para o desenvolvimento cognitivo da criança ao ofertar brinquedos, livros e oferecer oportunidades para a realização de atividades lúdicas e educativas. A família também pode oferecer suporte emocional através de um ambiente acolhedor, um bom relacionamento entre os pais e entre pais e filhos gera motivação para aprendizagem (PICCOLO et al., 2012).

Conforme Piccolo et al. (2012), no tópico "fatores que interferem na aprendizagem da leitura", crianças cujas famílias têm NSE mais baixo apresentam maiores dificuldades de aprendizagem devido a vários fatores tais como: renda familiar, a saúde emocional da mãe, a quantidade de pessoas que moram com a criança e tempo disponível dos pais com a criança.

Após compreender as características desta população, é importante pensar em serviços a serem oferecidos para este público a fim de minimizar os riscos de vulnerabilidade das crianças, para melhorar a qualidade de vida destas famílias. Tais como fortalecer políticas públicas de qualificação do processo educativo e de cuidado e atendimento às populações em situação de vulnerabilidade social e ambiental, sendo 
necessária uma rede de relacionamentos que funcione em prol do desenvolvimento das crianças e de suas famílias (ENRICONE; SALLES, 2011).

Diante deste estudo, foi possível compreender o quão complexo é quando se trata de dificuldades de aprendizagem, é preciso olhar além dos testes de desempenho da criança, é necessário avaliá-la como um todo, observando o mundo em que está inserida, sem fazer juízos de valor ou rotular a criança. Para então poder criar estratégias de facilitação para a sua aprendizagem.

\section{REFERÊNCIAS BIBLIOGRÁFICAS}

AARON, P. G.. Diagnosis of Reading Disabilities. School Psychology Review, 24(3), 45-60, 1995.

AIKENS, N. L.; BARBARIN, O. Socioeconomic Differences in Reading Trajectories: The Contribution of Family, Neighborhood, and School Contexts. Journal of Educational Psychology, 100(2), 235-251, 2008.

ANDERSON, P.. Assessment and development of executive function during childhood. Child Neuropsychology, 8, 71-82, 2002.

ANDRADA, E. G. C. et al. Fatores de risco e proteção para a prontidão escolar. Psicologia: Ciência e Profissão, 28(3), 536-547, 2008.

ANGELUCCI, C. B.; KALMUS, J.; PAPARELLI, R.; PATTO, M. H. S. O estado da arte da pesquisa sobre o fracasso escolar (1991-2002): um estudo introdutório.

Educação e Pesquisa, São Paulo, v. 30, n. 1, p. 51-72, jan./abr, 2004.

APA, American Psychological Association. DSM V: Manual diagnóstico e estatístico de transtornos mentais. Porto Alegre: Artmed, 2012.

ARÁN-FILIPPETTI, V.. Verbal fluency according to task type, time interval and socioeconomic status in school-aged children. Anales de Psicologia, 27(3), 816- 826, 2011.

ARDILA, A.; ROSSELLI, W.; MATUTE, E.; GUAJARDO, S.. The influence of the parents' educational level on the development of executive functions. Developmental Neuropsychology, 28(1), 539-560, 2005.

CARDOSO, C. O.; FONSECA, R. P. PENCE: Programa de Estimulação Neuropsicológica da Cognição em Escolares: ênfase nas funções executivas, 2016.

CARVALHO, A. M. P.. Baixo rendimento escolar: uma visão a partir do professor. In FUNAYAMA, C. A. R. (Org.). Problemas de aprendizagem: enfoque multidisciplinar. Campinas, SP: Alínea, 2000.

CORSO, H.. A representação infantil e a educação pré-escolar. Educação e realidade, Porto Alegre, v. 18, n. 1, p. 61-70, jan./jun, 1993.

Educação, Psicologia e Interfaces, Volume 2, Número 2, p. 7-28, Maio/Agosto, 2018.

ISSN: 2594-5343. DOI: https://doi.org/10.37444/issn-2594-5343.v2i2.93 
CORSO, H. V.; SPERB, T. M.; SALLES, J. F.. Leitura de palavras e de texto em crianças: efeitos de série e tipo de escola, e dissociações de desempenhos. Letras de Hoje, Porto Alegre, v. 48, n. 1, p. 81-90, jan./mar, 2013.

DAWNSON, P.; GUARE, R.. Executive Skills in children and adolescentes: a pratical guide to assessment and intervention. New York: The Guilford Press, 2010.

DIAS, N. M.; SEABRA, A. G.. PIAFEX: Programa de intervenção em autorregulção e Funções Executivas. Memnon edições científicas. São Paulo, 2013.

DUURSMA, E.; PAN, B. A.; E RAIKES, H. . Predictors and outcomes of low-income fathers reading with their toddlers. Early Childhood Research Quarterly, 23, 351$365,2008$.

ENRICONE, J. R. B; SALLES, J. F.. Família e Desempenho em Leitura/Escrita. Revista Semestral da Associação Brasileira de Psicologia Escolar e Educacional, SP. v. 15, n. 2, p. 199-210, jul./dez, 2011.

EVANS, M. A.; SHAW, D.; BELL, M.. Home literacy activities and their influence on early literacy skills. Canadian Journal of Experimental Psychology, 54, 65-75, 2000.

FERNALD, L. C. H.; WEBER, A.; GALASSO, E.; RATSIFANDRIHAMANANA, L.. Socioeconomic gradients and child development in a very low income population: evidence from Madagascar. Developmental Science, 14(4), 832-847, 2011.

FLETCHER, J. M.; LYONS, G. R.; BARNES, M. A.. Transtornos de aprendizagem: da identificação à intervenção. Porto Alegre: Artmed, 2007.

FONSECA, N. G.. A influência da família na aprendizagem da criança. São Paulo, 1999.

FOY, J. G.; MANN, V.. Home literacy environment and phonological awareness in preschool children: Differential effects for rhyme and phoneme awareness. Applied Psycholinguistics, 24, 59-88, 2003.

GAZZANIGA, M. S.; IVRY, R. B.; MANGUN, G. R.. Neurociência cognitiva. Porto Alegre: Artmed, 2016.

GOLDMANN, S.; WOLFE, M.. Text Comprehension: Models in Psychology. In:

SMELSER, N.; BALTES, P. International Encyclopedia of the Social \& Behavioral Sciences, Oxford: Elsevier, 2001. p. 15625-15631, 2001.

HACKMAN, D. A.; FARAH, M. J.. Socioeconomic status and the developing brain. Trends in Cognitive Sciences, 13(2), 65-73, 2009.

HACKMAN, D. A.; FARAH, M. J.; MEANEY, M. J.. Science and society: socioeconomic status and the brain: mechanistic insights from human and animal research. Nature Reviews Neuroscience, 11(9), 651-659, 2010.

HANEY, M.; HILL, J.. Relationship between parent-teaching activities and emergent literacy in preschool children. Early Child Development and Care, 174, 215- 228, 2004. 
HURKS, P. et al.. Developmental changes in semantic verbal fluency: analyses of word productivity as a function of time, clustering, and switching. Child Neuropsychology, $16,366-387,2010$.

IBGE - Instituto Brasileiro de Geografia e Estatística. Síntese dos Indicadores Sociais. A Educação melhora mas ainda apresenta desafios, 2008. Recuperado: 08 nov 2011. Disponível: http:// www.ibge.gov.br/home/presidencia/noticias/noticia_visualiza. php?id_noticia=1233\&id_pagina $=1$.

IBGE - Instituto Brasileiro de Geografia e Estatística. Síntese de indicadores sociais: uma análise das condições de vida da população brasileira , 2012. Disponível em: https://www.ibge.gov.br/estatisticas-novoportal/sociais/justica-e-seguranca/9221sintese-de-indicadores-sociais.html?edicao $=17068 \& \mathrm{t}=0$-que-e.

INEP, Instituto Nacional de Estudos e Pesquisas Educaionais Anísio Teixeira. Organização para Cooperação e Desenvolvimento Econômico, 2015. Retrieved February 2, 2015, from http://portal.inep.gov.br

KIRBY, J.; HOGAN, B.. Family Literacy Environment and Early Literacy Development. Exceptionality Education, Canada, v. 18, n. 3, p. 112-130, 2008.

KUO, A. A., FRANKE, T. M., REGALADO, M., E HALFON, M.. Parent report of reading to young children. Pediatrics, 113(6), 1944-1951, 2004.

LEYBAERT, J.; ALÉGRIA, J.; DELTOUR, J.; SKINKEL, R.. Aprender a ler: o papel da linguagem, da consciência fonológica e da escola. In: GRÉGOIRE, J.; PIÉRART, B. (Orgs.). Avaliação dos problemas de leitura: os novos modelos teóricos e suas implicações diagnósticas. Porto Alegre: Artes Médicas, 1997.

LEZAK, M. D.; HOWIESON, D. B.; LORING, D. W.. Neuropsychological Assessment. 4th ed, New York: Oxford University Press, 2004.

LIMA, M.. Desempenho em leitura de crianças de $4^{\circ}$ ano do ensino fundamental: fatores neuropsicológicos e ambientais. Dissertação de mestrado. Porto Alegre, RS, 2016.

MALLOY-DINIZ, L. F.; SEDO, M.; FLUENTES, D.; LEITE, W. B.. Neuropsicologia das funções executivas. In: D. FLUENTES; L. F. MALLOY-DINIZ; C.H.P.

CAMARGO; R.M. COSENZA, R. M (Orgs.) Neuropsicologia: Teoria e Prática. Porto Alegre: Artmed, 2008.

MARCHESI, A.; GIL, C. H.. Fracasso escolar: uma perspectiva multicultural. Porto Alegre: Artmed, 2004.

MCLOYD, V. C. . Socioeconomic disadvantage and child development. The American Psychologist, 53 (2), 185-204, 1998. Retrieved from http://www.ncbi.nlm.nih.gov/pubmed/9491747.

MELTZER, L.. Promoting executive functions in the classroom. New York, NY: The Guilford Press, 2010.

MENDES, A. V.; LOUREIRO, S. R.; CRIPPA, J. A. S.. Depressão materna e a saúde mental de escolares. Revista de Psiquiatria Clínica, 35(5), 178-186, 2008.

Educação, Psicologia e Interfaces, Volume 2, Número 2, p. 7-28, Maio/Agosto, 2018.

ISSN: 2594-5343. DOI: https://doi.org/10.37444/issn-2594-5343.v2i2.93 
MIRANDA, M. C.; MUSZKAT, M.. Neuropsicologia do desenvolvimento. In: ANDRADE, V. M.; SANTOS, F. H.; ORLANDO, F. A. B. Neuropsicologia hoje. São Paulo: Artes Médicas, 2004.

MUSSEN, P. H.. O desenvolvimento psicológico da criança. $5^{\text {a }}$ edição. Rio de Janeiro, 1970.

NOBLE, K. G.; NORMAN, M. F.; FARAH, M. J.. Neurocognitive correlates of socioeconomic status in kindergarten children. Developmental Science, 8(1), p. 74-87, 2005.

PICCOLO, L. R. . Relações entre variáveis psicossociais e cognitivas e o desempenho em leitura em crianças de uma coorte populacional. Dissertação de mestrado. Universidade Federal do Rio Grande do Sul. Porto Alegre/RS, 2010.

PICCOLO, L. R. et al.. Variáveis psicossociais e Desempenho em Leitura de Crianças de Baixo Nível Socioeconômico. Psicologia: Teoria e Pesquisa, Vol. 28 n. 4, pp. 389398, 2012.

PINTO, I. D.; PADOVANI, F. H. P.; LINHARES, M. B. M.. Ansiedade e depressão materna e relatos sobre o bebê prematuro. Psicologia: Teoria e Pesquisa, 25(1), 75-83, 2009.

PRUEZA, J. R.; FONSECA, R. P. Cena - Programa de capacitação de educadores sobre neuropsicologia da aprendizagem com ênfase em funções executivas e atenção.

Sinopsys, Novo Hamburgo: RS, 2016.

RADKE-YARROW, M.; ZAHN-WAXLER, C. Research on affectively ill parents: Some considerations for theory and research on normal development. Development and Psychopathology, 2, 349-366, 1990.

RHOADES, B.; GREENBERG, M.; LANZA, S.; BLAIR, C.. Demographic and familial predictors of early executive function development: contribution of a personcentered perspective. Journal of Experimental Child Psychology, 108, 638-662, 2011.

SALLES, J. F.; PARENTE, M. A. M. P.; FREITAS, L. B. L. Leitura/escrita de crianças: comparações entre grupos de diferentes escolas públicas. Paidéia. 2010, Vol. 20, No. 47, 335-344, 2010.

SBICIGO, J. B.; ABAID, J. L. W.; DELL'AGLIO, D. D.; SALLES, J. F.. Nível socioeconômico e funções executivas em crianças/adolescentes: revisão Sistemática. Arquivos Brasileiros de Psicologia; Rio de Janeiro, 65 (1): 51-69, 2013.

SÉNÉCHAL, M.; LEFREVE, J. A.. Parental involvement in the development of children's reading skill: A five-year longitudinal study. Child Development, 73(2), 445-460, 2002.

SKIBBE, L. E.; JUSTICE, L. M.; ZUCKER, T. A.; MCGINTY, A. S.. Relations among maternal literacy beliefs, home literacy practices, and the early literacy skills of preschoolers with specific language impairment. Early Education and Development, 19, 68-88, 2008.

STERNBERG, R. J.; GRIGORENKO, E. L. Our labeled children. Cambridge:Perseu Publishing, 2000. 


\section{Credenciais das autoras}

SILVA, Thalita Rodrigues. Graduação em Psicologia pela Universidade do Estado de Minas Gerais (UEMG - Campus Divinópolis). Especialização em Neuropsicologia pela Faculdade de Ciências Médicas de Minas Gerais (FCMMG). E-mail: rds.thalita@gmail.com

SILVA, Julia Beatriz Lopes. É pesquisadora colaboradora do Laboratório de Neuropsicologia do Desenvolvimento (LND) da Universidade Federal de Minas Gerais e Professora Adjunta do departamento de Psicologia da Universidade Federal de Minas Gerais (UFMG). E-mail: silvajbls@gmail.com

Como citar este artigo (Formato ABNT): SILVA, Thalita Rodrigues; SILVA, Julia Beatriz Lopes. A influência do nível socioeconômico na aprendizagem da leitura. Educação, Psicologia e Interfaces, v. 2, n.2, p. 7-28, 2018. DOI: https://doi.org/10.37444/issn-2594-5343.v2i2.93

Recebido: 04/02/2018.

Aceito: 26/04/2018. 